\title{
Palmitoylated proteins: purification and identification
}

\author{
Junmei Wan ${ }^{1}$, Amy F Roth ${ }^{1}$, Aaron O Bailey ${ }^{2,3} \&$ Nicholas G Davis $^{1}$ \\ ${ }^{1}$ Department of Pharmacology, Wayne State University School of Medicine, Detroit, Michigan 48201, USA. ${ }^{2}$ Department of Cell Biology, The Scripps Research Institute, \\ La Jolla, California 92037, USA. ${ }^{3}$ Present address: Program in Cell and Developmental Biology, University of Virginia School of Medicine, Charlottesville, Virginia 22908, \\ USA. Correspondence should be addressed to N.G.D. (ndavis@med.wayne.edu).
}

Published online 21 June 2007; doi:10.1038/nprot.2007.225

This proteomic protocol purifies and identifies palmitoylated proteins (i.e., S-acylated proteins) from complex protein extracts. The method relies on an acyl-biotinyl exchange chemistry in which biotin moieties are substituted for the thioester-linked protein acyl-modifications through a sequence of three in vitro chemical steps: (i) blockade of free thiols with N-ethylmaleimide; (ii) cleavage of the Cys-palmitoyl thioester linkages with hydroxylamine; and (iii) labeling of thiols, newly exposed by the hydroxylamine, with biotin-HPDP (Biotin-HPDP-N-[6-(Biotinamido)hexyl]-3'-(2'-pyridyldithio)propionamide. The biotinylated proteins are then affinity-purified using streptavidin-agarose and identified by multi-dimensional protein identification technology (MuDPIT), a high-throughput, tandem mass spectrometry (MS/MS)-based proteomic technology. MuDPIT also affords a semi-quantitative analysis that may be used to assess the gross changes induced to the global palmitoylation profile by mutation or drugs. Typically, 2-3 weeks are required for this analysis.

\section{INTRODUCTION}

Protein palmitoylation (or, more correctly, protein S-acylation) is the thioesterification of fatty acyl moieties, typically the 16-carbon palmitoyl moiety, to selected protein cysteines. Similar to prenylation and myristoylation, and often in combination with these two lipidations, palmitoylation may serve to tether proteins to membrane cytosolic surfaces (for reviews, see refs. 1-3). Many signaling proteins, including key players in cancer and synaptic signaling, are palmitoylated. Notably, many $G$ proteins rely on palmitoylation for proper membrane-localized function, including $\mathrm{H}$ - and $\mathrm{N}$-Ras, some Rho proteins, as well as the $\alpha$ subunits of most heterotrimeric $G$ proteins. In distinction to prenylation and myristoylation, palmitoylation frequently also is found as a transmembrane (TM) protein modification. For the TM proteins, embedded in the membrane by its hydrophobic, bilayer-spanning TM domains, the addition of palmitoyl tethers seems superfluous. For these TM palmitoylproteins (PPs), a palmitoylation role in targeting to raft-like membrane domains often is invoked; the saturated palmitoyl moiety should increase affinity for the cholesteroland sphingolipid-rich, liquid-ordered membrane domain. Another unique feature of protein palmitoylation that excites interest is its reversibility. The regulated addition and removal of palmitoyl tethers provides an attractive mechanism for controlling membrane and/or raft association. Such control of targeting by reverse palmitoylation, however, has yet been demonstrated only for a small handful of PPs.

The protocol detailed below, first published as part of a global analysis of palmitoylation in the yeast Saccharomyces cerevisiae $^{4,5}$, purifies and identifies the subset of proteins that are palmitoylated from highly complex protein extracts. This $\mathrm{PP}$ purification is a proteomic extrapolation of the acyl-biotinyl exchange (ABE) chemistry of Drisdel and Green, an in vitro method that substitutes biotinyl moieties for the thioester-linked palmitoyl modifications ${ }^{6}$. The ABE-generated biotinylated proteins can then be affinity-purified using streptavidinagarose and identified by proteomic mass spectrometry (MS). ABE comprises a sequence of three chemical steps: (i) an exhaustive blockade of free thiols with $N$-ethylmaleimide (NEM); (ii) hydroxylamine treatment to release thioester-linked palmitoyl moieties, restoring the modified cysteine to thiols, (iii) which are then biotinylated using a thiol-reactive biotinylation reagent (Fig. 1). As the biotinylation reagent, we have opted to use biotin-HPDP, which has several advantages over the biotin-BMCC originally used by Drisdel and Green ${ }^{6}$. Biotin-HPDP, which disulfide bonds to the thiols, is more thiol-specific; furthermore, it facilitates an easy



Figure 1 | Schematic of the proteomic acyl-biotinyl exchange methodology. 
release of proteins bound to the streptavidin-agarose affinity matrix, through $\beta$-mercaptoethanol-mediated cleavage of the biotinCys linkage.

An important control, included in all our analyses, is the processing of an equal portion of the initial protein extract through a parallel $\mathrm{ABE}$ protocol that omits the hydroxylamine thioester-cleavage step. In the absence of hydroxylamine, palmitoyl modifications should not be removed and PPs should not be biotinylated or purified. However, owing either to inappropriate biotinylation or non-specific streptavidin-agarose binding, some proteins do end up purified into the minus-hydroxylamine (-HA) control sample (Fig. 2). These -HA sample proteins also are nonspecifically purified into the experimental plus-hydroxylamine (+HA) sample and thus represent a source of false-positive identifications. In addition to contaminant proteins, there also is a clear set of + HA sample proteins that show the HA-dependent purification indicative of likely PP status (Fig. 2). The challenge is to distinguish true PPs from the non-specifically purified contaminant proteins; in the protocol below, this is accomplished using a quantitative MS analysis that compares each protein's representation in parallel $+\mathrm{HA}$ and $-\mathrm{HA}$ samples.

\section{Multi-dimensional protein identification technology analysis}

HA samples $(+$ and - ) are analyzed by multi-dimensional protein identification technology (MuDPIT), a high-throughput, tandem MS-based proteomic technology developed for the analysis of highly complex protein samples ${ }^{7,8}$. MuDPIT is distinguished by its orthogonal 2D peptide separation, which occurs before, but in-line with, the tandem MS. Following exhaustive proteolysis of the protein samples, the peptides are fractionated from one another both by charge and by hydrophobicity, continuously eluting into the tandem MS for sequencing over an 8-12 h timeframe. A single typical MuDPIT run of a complex sample may sequence tens of thousands of peptides that link to hundreds or thousands of database proteins. As MuDPIT is a highly complex technology that utilizes expensive MS instrumentation, it is assumed that the researcher embarking on the protocol below will first establish a collaboration with an experienced scientist with ample experience in running MuDPIT.

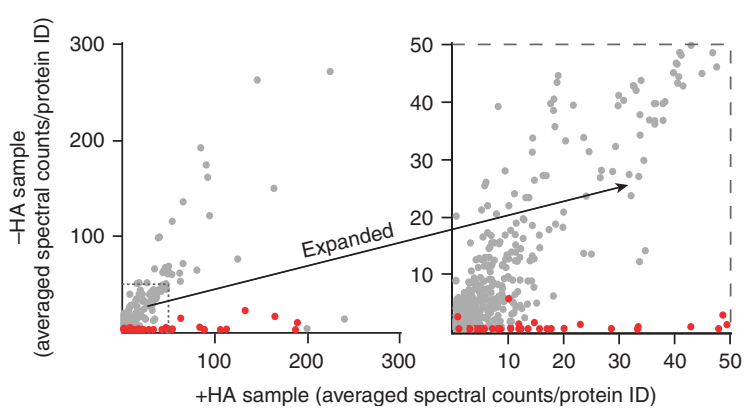

Figure 3 | An $x, y$-scatter plot depiction of yeast proteins identified by multi-dimensional protein identification technology (MuDPIT) of plus- and minus-hydroxylamine (HA) samples. Each of the 1,558 proteins identified by MuDPIT analysis in our yeast proteomic analysis is plotted as averaged, normalized $+\mathrm{HA}$ sample spectral counts ( $x$-coordinate) against averaged, normalized $-\mathrm{HA}$ sample spectral counts ( $y$-coordinate) ${ }^{4}$. The palmitoyl proteins (PPs), including both the 15 PPs that were known to be palmitoylated at the outset of this analysis and the 35 PPs newly identified and confirmed by this analysis, are shown in red. The right-hand panel shows



Figure 2 | SDS-polyacrylamide gel electrophoresis (SDS-PAGE) of purified parallel plus- and minus-hydroxylamine (HA) yeast samples just before proteomic multi-dimensional protein identification technology (MuDPIT) analysis. A protein extract derived from total yeast membranes was split in half and purified through the $+\mathrm{HA}$ and $-\mathrm{HA}$ acyl-biotinyl exchange (ABE) protocols in parallel. After the final streptavidin-agarose purification, a comparison was made of $1 \%$ of each of the two samples by SDS-PAGE and silver-staining. Presumptive contaminant proteins, showing HA-independent purification, are marked to the right by hash marks, while the candidate PPs, which show HA-dependent purification, are indicated with arrows. Reprinted with permission from ref. 4 .

MuDPIT, in addition to providing comprehensive sample coverage, also affords a crude but facile quantitative analysis. Unlike other quantitative proteomic approaches, which typically require differential labeling of samples with heavy and light isotopes and complex downstream data analysis ${ }^{9}$, MuDPIT allows a crude quantitation which is based upon a parameter that is part of the standard MuDPIT dataset: the spectral count number. The spectral count number is the number of sequenced peptides that link to each identified protein. Within a single MuDPIT run, abundant sample proteins often are independently re-identified multiple times, both through identification of the protein's different component peptides and through iterative re-identification of the same peptide eluting in multiple fractions owing to peak broadening within the initial chromatographic separations. The spectral count number, which includes these redundant peptide identifications, has been demonstrated to be a useful metric for comparing a protein's relative abundance among samples ${ }^{10}$.

Quantitation based on spectral count has proven critical both for identifying PPs and for analyzing the effects of treatments that perturb palmitoylation ${ }^{4}$. Distinguishing PPs from the contaminant protein background is based on comparing spectral counts for each protein from parallel $+\mathrm{HA}$ and - HA samples. Non-specifically purified contaminant proteins show roughly equivalent abundances in +HA and - HA samples, whereas the PPs show exclusive, or substantially higher, +HA sample abundances. Figure 3, a spectral count analysis of the proteins identified from $+\mathrm{HA}$ and -HA ABE samples from the yeast $S$. cerevisiae, illustrates the utility of the spectral count parameter. All 1,558 yeast proteins identified from MuDPIT analyses of four parallel $+\mathrm{HA}$ and $-\mathrm{HA}$ samples are plotted. Each protein (each dot) is plotted as averaged + HA sample spectral counts on the $x$-axis versus averaged -HA sample spectral counts on the $y$-axis. Of the 1,558 total yeast proteins identified, the vast majority are not palmitoylated, but rather contaminant proteins, showing significant representations in both $+\mathrm{HA}$ and -HA samples and trending, therefore, toward the $x, y$-diagonal (Fig. 3). These contaminant proteins tend to be proteins of known 
yeast cell high abundance ${ }^{11}$. Many of the PPs (red dots), a group that includes both previously known PPs and PPs newly identified by this analysis, are detected only from the +HA samples and thus map onto the $x$-axis (Fig. 3). Other PPs map near, but not directly on top of, the $x$-axis (Fig. 3), reflecting a + HA sample bias that is not fully exclusive. This second class of PPs, which are also detected at low levels from - HA samples, would be overlooked by a nonquantitative analysis that simply subtracts the list of - HA proteins from the list of $+\mathrm{HA}$ proteins. In addition to identifying 12 of the 15 known yeast PPs, our analysis also identified and confirmed palmitoylation for an additional 35 proteins $s^{4}$. The identified PPs encompassed all the known types of PPs-proteins that tether to membranes solely through palmitoylation, proteins that are palmitoylated in addition to being also either $\mathrm{N}$-terminally myristoylated or C-terminally prenylated; as well as many TM PPs.

Our spectral count-based approach, in addition to allowing PPs to be distinguished from co-purifying contaminants, may be used to assess the global changes in palmitoylation that can be induced by drugs or mutation. The power of such an approach is illustrated by our recent mapping of the yeast PPs to their cognate palmitoylation enzymes ${ }^{4}$. The palmitoyl proteome of a wild-type yeast strain was compared to the proteomes of mutant strains deleted for the genes encoding the seven members of the newly identified DHHC (Asp-His-His-Cys) protein acyl transferase (PAT) family ${ }^{12-14}$. PP substrates of a particular PAT are expected to be lost from the palmitoyl proteome of strains deficient for that PAT; thus, substrates are highlighted by their absence from the DHHC deletion strain palmitoyl proteome (Fig. 4). This analysis finds not only PPs that fully drop out but also PPs with partial under-representations, indicative of a partial requirement for the deleted PAT. Analysis of strains multiply deleted for the different DHHC genes allows the overlapping specificity relationships among the different DHHC PATs to be discerned. Many of the enzyme-substrate relationships uncovered in this comparative proteomic analysis have indeed been confirmed in targeted testings of the palmitoylation of individual PPs in different DHHC mutant backgrounds ${ }^{4}$. In principle, this approach should allow global changes in palmitoylation to be monitored in response to a wide variety of perturbants and stimuli-e.g., hormonal signals, drugs and changes in growth conditions. Indeed, the mammalian DHHC PAT family is an important set of future drug targets with possible utility in the treatment of cancer and other diseases. Recently, a first generation of compounds with inhibitory effects on palmitoylation has been developed ${ }^{15}$. Comparing the profiles from drug-treated cells with those of mutants deficient in the individual PATs should provide a means of mapping drugs to their target PATs.

The protocol that follows should be applicable to tissue from any source organism with the available sequence data to allow MSbased protein identification. As indicated above, this protocol has been used in yeast both to identify many new PPs and to map substrate PPs with their cognate modifying DHHC PAT ${ }^{4}$. Recently, we have applied the same approach to the analysis of various mammalian palmitoyl proteomes-from mouse or rat whole brain, and from primary cultures of embryonic rat brain neurons. Similar to our yeast analyses ${ }^{4}$, our initial mammalian ABE purifications also readily pull out a mix of both known and new PPs; indeed, our analysis of rat embryonic neurons identifies 24 known PPs from among the 100 top-scoring proteins (R. Kang, J.W., A.O.B., J. Yates, N.G.D. and A. El-Husseini, unpublished results). For

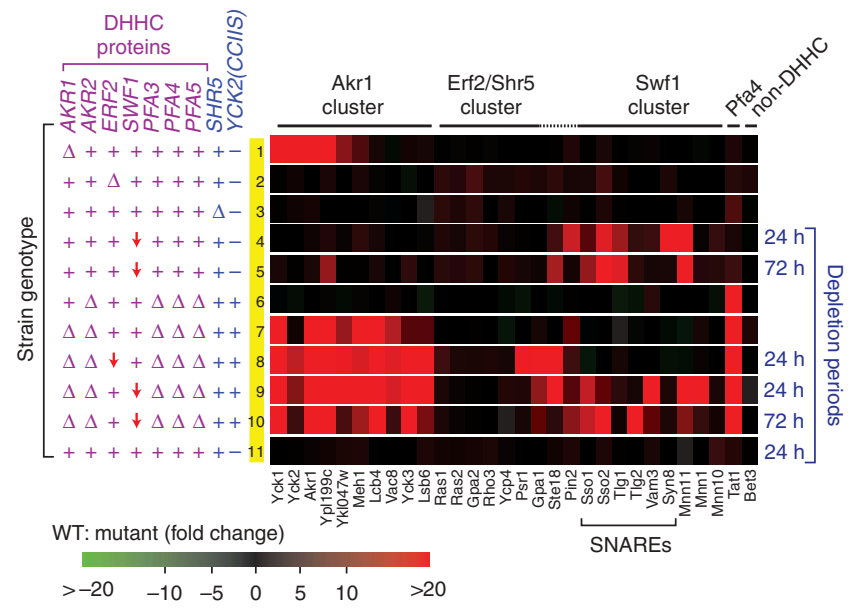

Figure 4 | Colorimetric depiction of palmitoylation changes seen in strains deficient for the different yeast DHHC protein acyl transferases (PATs). Plushydroxylamine (HA) samples purified from 11 different wild-type and mutant yeast strains were analyzed by multi-dimensional protein identification technology (MuDPIT) with the 30 most prominent palmitoyl proteins (PPs) (listed at the bottom) being compared for relative abundance with the spectral count metric. Wild-type:mutant spectral count ratios for each protein were converted to color, with proteins showing 20 -fold or greater mutant sample under-representations shown in red, and proteins with intermediate under-representations depicted by intermediate red shadings (for details on the colorimetric conversion, see ref. 4). Relevant strain genotypes are indicated on the left: for each tested strain, the seven different yeast DHHC PAT-encoding genes are indicated as being wild-type $(+)$, deleted $(\Delta)$ or replaced by a conditional GAL1-driven depletion allele strain with the gene encoding the indicated DHHC PAT fully deleted $(\Delta)$ or, in a few cases, depletion of the indicated PAT was induced via glucose-mediated repression of a GAL1-driven PAT allele (downward arrow; glucose depletion periods are indicated at right). Note that for many PPs, palmitoylation is significantly blocked only in strains concomitantly mutated for multiple DHHC PATs, presumably indicating substantial specificity overlaps among the seven different DHHC PATs. Reprinted with permission from ref. 4. DHHC.

both the yeast and mammalian analyses, starting samples are typically scaled to approximately $10 \mathrm{mg}$ of starting total protein, although successful analyses have also been performed on samples that start with as little as $2 \mathrm{mg}$ of total protein. The initial steps of the protocol describe the preparation of starting protein extracts both from a yeast cell culture and from mouse whole brain. For other source tissues, initial homogenization may have to be varied appropriately. However, protocol steps subsequent to these initial homogenization steps should be identical to those detailed below.

The protocol is divided into the following sub-sections: (i) preparation of the starting protein extracts (the preparation of lysates from both yeast cultures and mouse brain as well as an optional crude membrane purification step to enrich for PPs is described); (ii) ABE; (iii) affinity-purification of biotinylated proteins; (iv) proteolysis and preparation of samples for MuDPIT analysis; (v) MuDPIT analysis (it is assumed that the MuDPIT analysis will be done collaboratively with a proteomics facility well versed in the procedure; consequently, the MuDPIT portion of the protocol is presented in overview fashion, with the particular parameters that may be unique to our analyses of palmitoylation indicated. For an excellent step-by-step MuDPIT protocol detailing both the set-up and running of MuDPIT, the reader is referred to ref. 16); (vi) spectral count-based quantitation. 
PROTOCOL

\section{MATERIALS}

REAGENTS

- Pepstatin (Sigma)

- Antipain (Sigma)

- Chymostatin (Sigma)

- Leupeptin (Sigma)

- Triton X-100 10\% solution (Anatrace Anapoe, cat. no. X-100)

- NEM (Pierce, cat. no. PI 23030)

- Hydroxylamine (Sigma)

- HPDP-biotin (Pierce, cat. no. PI 21341)

-Streptavidin-agarose (Pierce, cat. no. PI 20349)

- $\beta$-Mercaptoethanol (Fisher, cat. no. BP176)

- Tris(2-carboxyethyl)phosphine (TCEP; Sigma)

- Iodoacetamide (Sigma)

- Endoproteinase Lys-C (Roche)

- Glucose

- Peptone

- Yeast extract

- Trypsin (Roche)

- Reversed-phase resins-Aqua C18 (3- $\mu \mathrm{m}$ beads, 100-Å pores) and Aqua C18

(5- $\mu \mathrm{m}$ beads, $300-\AA ̊$ pores) (Phenomenex)

- Strong cation exchange resin-Luna SCX $(5-\mu \mathrm{m}$ beads, $100-\AA ̊ \AA$ pores;

Phenomenex)

- Acetonitrile (HPLC grade)

- Ammonium acetate

- Formic acid

- $1 \mathrm{M}$ Tris/Cl, pH 7.4

$\cdot 1 \mathrm{M}$ Tris/Cl, pH 8.5

$\cdot 0.5 \mathrm{M}$ EDTA, pH 8.0

$\cdot 1 \mathrm{M}$ NEM in ethanol $\triangle$ CRITICAL Prepare fresh for every experiment; store on ice.

$\cdot 1 \mathrm{mg} \mathrm{ml}^{-1}$ pepstatin in methanol $\Delta$ CRITICAL Store at $-20{ }^{\circ} \mathrm{C}$.

$\cdot 10 \mathrm{mg} \mathrm{m}^{-1}$ leupeptin in water $\triangle$ CRITICAL Store at $-20^{\circ} \mathrm{C}$.

$\cdot 10 \mathrm{mg} \mathrm{ml}^{-1}$ antipain in DMSO $\Delta$ CRITICAL Store at $-20^{\circ} \mathrm{C}$.

- $10 \mathrm{mg} \mathrm{ml}^{-1}$ chymostatin in ethanol $\Delta$ CRITICAL Store at $-20{ }^{\circ} \mathrm{C}$.

- 0.1 M phenylmethanesulfonyl fluoride (PMSF; Sigma, cat. no. P7626) in ethanol $\triangle$ CRITICAL Store at $4{ }^{\circ} \mathrm{C}$.

- 1 M hydroxylamine, pH 7.4 $\Delta$ CRITICAL Prepare fresh for each experiment; store on ice.

- $50 \mathrm{mM}$ HPDP-biotin in DMSO $\triangle$ CRITICAL Store at $-20^{\circ} \mathrm{C}$; solution may become somewhat cloudy after $-20^{\circ} \mathrm{C}$ storage.

- $4 \mathrm{mM}$ HPDP-biotin in $\mathrm{N}, \mathrm{N}$-dimethyl formamide $\triangle$ CRITICAL Dilute from

$50 \mathrm{mM}$ stock just before use; keep on ice until use.

- $100 \mathrm{mM}$ TCEP $\Delta$ CRITICAL Store at $-20^{\circ} \mathrm{C}$.

$\cdot 1 \mathrm{M} \mathrm{CaCl}_{2}$

- $0.5 \mathrm{M}$ iodoacetamide $\Delta$ CRITICAL Prepare fresh for every experiment; store on ice.

- MuDPIT buffer A ( $5 \%$ acetonitrile, $0.1 \%$ formic acid)

- MuDPIT buffer B ( $80 \%$ acetonitrile, $0.1 \%$ formic acid)

- MuDPIT buffer C (500 mM ammonium acetate, $5 \%$ acetonitrile, $0.1 \%$

formic acid)

\section{EQUIPMENT}

- Coffee grinder (Krups, cat. no. F2037051)

- Sorvall (Dupont) RC-5B centrifuge with GSA and HS-4 rotors

-50-ml disposable centrifuge tubes (Sarstedt, cat. no. 62.547.205)

-12-ml screw-cap centrifuge tube (Sarstedt, cat. no. 60.540)

-1.5-ml screw-cap centrifuge tube (Sarstedt, cat. no. 72.692)

- IKA tissue homogenizer (T25 basic; Ultra Turrax, cat. no. T25BS1) with

S25N-8G blade attachment

-Sonicator (Sonic Dismembrator Model 500; Fisher Scientific) with micro-probe (Branson model 1020)

- Ultra-centrifuge and fixed angle rotor (optional)-e.g., Beckman L8-M ultra-centrifuge with Type 80Ti rotor (Beckman)
- Tube rotator (Thermolyne Labquake, cat. no. 400110)

- Micro-centrifuge

- Tube rocker (Thermolyne Vari-Mix, cat. no. M48725)

- LTQ ion-trap mass spectrometer (Thermo-Finnigan)

- HPLC (Agilent)

- Fast computer system for analysis of tandem mass spectra and for database searching

- MuDPIT microcapillary chromatography set-up (see EQUIPMENT SETUP)

- Microfilter assembly (UpChurch Scientific, Oak Harbour, WA) (see

EQUIPMENT SETUP)

REAGENT SETUP

YPD ( $1 \%$ yeast extract, $2 \%$ peptone, $2 \%$ glucose) For 11 , dissolve $10 \mathrm{~g}$ yeast extract, 20 g peptone in $960 \mathrm{ml}$ water. Autoclave to sterilize, then add $40 \mathrm{ml}$ of sterile $50 \%$ glucose.

Lysis buffer (LB; $150 \mathrm{mM} \mathrm{NaCl}, 50 \mathrm{mM}$ Tris, $5 \mathrm{mM}$ EDTA, pH 7.4) For $100 \mathrm{ml}$, combine $3 \mathrm{ml} 5 \mathrm{M} \mathrm{NaCl}, 5 \mathrm{ml}$ Tris/Cl pH 7.4, $1 \mathrm{ml} 0.5 \mathrm{M}$ EDTA and $91 \mathrm{ml}$ water. Supplement with necessary components (e.g., Triton X-100, NEM, PI) to the concentrations indicated in the protocol.

$100 \times$ protease inhibitors (PIs) Combine $25 \mu \mathrm{g} \mathrm{ml}^{-1}$ each of pepstatin, leupeptin, antipain and chymostatin. For $1 \mathrm{ml}$, combine $250 \mu \mathrm{l} 1 \mathrm{mg} \mathrm{ml}^{-1}$ pepstatin, $25 \mu 10 \mathrm{mg} \mathrm{ml}^{-1}$ leupeptin, $25 \mu 10 \mathrm{mg} \mathrm{ml}^{-1}$ antipain, $25 \mu \mathrm{l}$ $10 \mathrm{mg} \mathrm{ml}^{-1}$ chymostatin and $675 \mu \mathrm{l}$ ethanol.

4\% SDS buffer (4SB; 4\% SDS, 50 mM Tris, 5 mM EDTA, pH 7.4) For $10 \mathrm{ml}$ combine $4 \mathrm{ml} \mathrm{10 \%} \mathrm{SDS,} 0.5 \mathrm{ml} 1 \mathrm{M}$ Tris/Cl pH 7.4, $0.1 \mathrm{ml} 0.5 \mathrm{M}$ EDTA and $5.4 \mathrm{ml}$ water. Supplement with necessary components (e.g., NEM) to the concentrations indicated in the protocol.

+HA buffer (0.7 M hydroxylamine, $1 \mathrm{mM}$ HPDP-biotin, $0.2 \%$ Triton X-100, 1 mM PMSF, $1 \times$ PI pH 7.4) For $10 \mathrm{ml}$, combine $2.5 \mathrm{ml} 4 \mathrm{mM}$ HPDP-biotin, $0.2 \mathrm{ml} 10 \%$ Triton X-100, $0.1 \mathrm{ml} \mathrm{100 \times} \mathrm{PI,} 0.1 \mathrm{ml} 0.1 \mathrm{M}$ PMSF, $7 \mathrm{ml} 1 \mathrm{M}$ hydroxylamine $\mathrm{pH} 7.4$, and $0.1 \mathrm{ml}$ water.

-HA buffer (50 mM Tris, $1 \mathrm{mM}$ HPDP-biotin, 0.2\% Triton X-100, $1 \mathrm{mM}$ PMSF, $1 \times$ PI, pH7.4) For $10 \mathrm{ml}$, combine $2.5 \mathrm{ml} 4 \mathrm{mM}$ HPDP-biotin, $0.5 \mathrm{ml}$ Tris/Cl pH 7.4, $0.2 \mathrm{ml} 10 \%$ Triton X-100, $0.1 \mathrm{ml} \mathrm{100 \times} \mathrm{PI,} 0.1 \mathrm{ml} 0.1 \mathrm{M}$ PMSF and $6.6 \mathrm{ml}$ water.

Low HPDP-biotin buffer ( $150 \mathrm{mM} \mathrm{NaCl}, 50 \mathrm{mM}$ Tris, $5 \mathrm{mM}$ EDTA, $0.2 \mathrm{mM}$ HPDP-biotin, 0.2\% Triton X-100, 1 mM PMSF, $1 \times$ PI, pH 7.4) For $10 \mathrm{ml}$, combine $0.5 \mathrm{ml} 4 \mathrm{mM}$ HPDP-biotin, $0.3 \mathrm{ml} 5 \mathrm{M} \mathrm{NaCl}, 0.5 \mathrm{ml}$ Tris/Cl pH 7.4, $0.2 \mathrm{ml} 10 \%$ Triton X-100, $0.1 \mathrm{ml} 0.5 \mathrm{M}$ EDTA, $0.1 \mathrm{ml} 100 \times \mathrm{PI}, 0.1 \mathrm{ml} 0.1 \mathrm{M}$ PMSF and $8.2 \mathrm{ml}$ water.

2\% SDS buffer (2SB; 2\% SDS, $50 \mathrm{mM}$ Tris, $5 \mathrm{mM}$ EDTA, pH 7.4) For $10 \mathrm{ml}$, combine $2 \mathrm{ml} \mathrm{10 \%} \mathrm{SDS,} 0.5 \mathrm{ml} 1 \mathrm{M}$ Tris/Cl pH 7.4, $0.1 \mathrm{ml} 0.5 \mathrm{M}$ EDTA and $7.4 \mathrm{ml}$ water.

Proteolysis buffer (8 M urea, 0.1 M Tris, pH 8.5) For $100 \mathrm{ml}$, dissolve $6 \mathrm{~g}$ urea in $10 \mathrm{ml} 1 \mathrm{M}$ Tris/Cl pH 8.5, with water added to volume.

EQUIPMENT SETUP

MuDPIT microcapillary chromatography set-up (See ref. 16 for additional details). Desalting column is fused silica capillary column $(250-\mu \mathrm{m}$ i.d., $365-\mu \mathrm{m}$ o.d.; Agilent) filled with $4 \mathrm{~cm}$ of Aqua C18 RP resin ( 5 - $\mu \mathrm{m}$ beads, $300-\AA$ pores; Phenomenex, Torrance, CA).

Microfilter assembly Analytical microcapillary column (100- $\mu \mathrm{m}$ i.d., with laser-pulled 5-mm orifice) packed with $3 \mathrm{~cm}$ of Luna SCX resin $(5-\mu \mathrm{m}$ beads, 100-Å pores; Phenomenex) above $9 \mathrm{~cm}$ Aqua C18 RP resin (3- $\mu \mathrm{m}$ beads, $300-\AA$ pores; Phenomenex).

Software Xcalibur (Thermo-Finnigan), PARC, SEQUEST (ThermoFinnigan), Excel (Microsoft), DTASelect v1.9. Note: Although we have primarily relied on DTASelect v1.9 for our analyses, the newer version 2.0 utilizes an improved method for evaluating data quality that compares the number of database matches to the number of matches obtained from a decoy database in which protein sequences are reversed. By monitoring the frequency of matches to the decoy database, correlation coefficients and $\Delta \mathrm{C}_{n}$ cut-off value are automatically adjusted ad hoc to generate a dataset with the desired false-positive rate (D. Cociorva and J. Yates, personal communication).

\section{PROCEDURE}

\section{Preparation of starting protein extracts}

1) Tissue homogenization and cell lysis. Conditions are described for preparation of lysates from either yeast cultures (option A) or freshly resected mouse brain (option B). For other source materials, homogenization conditions may need to be altered. 


\section{(A) Yeast lysates}

(i) Collect by centrifugation $\left(4,000 \mathrm{~g}, 5 \mathrm{~min}, 4^{\circ} \mathrm{C}\right.$ in Sorvall GSA rotor) a 1-l late log-phase culture $\left(A_{600}=1.0 ; 1.5 \times 10^{7} \mathrm{ml}^{-1}\right)$ of YPD-grown yeast.

(ii) Re-suspend cell pellets in $40 \mathrm{ml}$ ice-cold LB with $10 \mathrm{mM} \mathrm{NEM,} \mathrm{2 \times} \mathrm{PI,} 2$ mM PMSF. Transfer to a 50-mL disposable centrifuge tube. Re-pellet cells $\left(5,000 \mathrm{~g}, 5 \mathrm{~min}, 4^{\circ} \mathrm{C}\right)$.

(iii) Aspirate buffer and freeze cell pellet on dry ice.

- PAUSE POINT Frozen cell pellet may be stored for several days at $-80^{\circ} \mathrm{C}$.

(iv) Cell lysis. Break open cell pellet-containing centrifuge tubes with one sharp hammer blow. Rapidly transfer the frozen cell pellet to the Krups coffee grinder, together with chunks of dry ice estimated to correspond to one-third of the cell pellet volume. Grind for a total of approximately $30 \mathrm{~s}$ (as grinding reaches completion, tone of grinder transitions to higher-pitch whine; continue for an additional $10 \mathrm{~s}$ ). Scoop cell lysate (viscous, partially frozen slurry) into cold 50-ml centrifuge tube. Incubate, unlidded, at $0{ }^{\circ} \mathrm{C}$, to allow residual dry ice to sublimate.

(v) Add $10 \mathrm{ml}$ of cold LB with $10 \mathrm{mM}$ NEM, 1× PI, 1 mM PMSF.

\section{(B) Mouse brain homogenate}

(i) Flash-freeze freshly resected mouse brain in liquid nitrogen.

PAUSE POINT Brain may be stored for several days at $-80{ }^{\circ} \mathrm{C}$.

(ii) Transfer frozen brain to ice-cold 12-ml centrifuge tube. Add $1 \mathrm{ml}$ of ice-cold LB with $10 \mathrm{mM} \mathrm{NEM}, 2 \times$ PI, and $2 \mathrm{mM}$ PMSF. Homogenize for 2 min at 24,000 r.p.m. using an IKA tissue homogenizer with S25N-8G blade attachment.

(iii) To further homogenize, sonicate, using micro-probe, with ten duty cycles of $1 \mathrm{~s} 0 \mathrm{~N}$ and $2 \mathrm{~s}$ OFF.

2) (Optional) Collect total membranes by high-speed centrifugation $\left(200,000 \mathrm{~g}, 30 \mathrm{~min}, 4^{\circ} \mathrm{C}\right)$ in fixed-angle rotor. This membrane purification is intended to enrich for PPs.

3| (Optional) Re-suspend membrane pellet in $3 \mathrm{~mL}$ LB buffer with $10 \mathrm{mM} \mathrm{NEM}, 1 \times$ PI and $1 \mathrm{mM}$ PMSF.

4| Detergent solubilization. Add Triton X-100 to $1.7 \%$. Incubate with end-over-end rotation at $4{ }^{\circ} \mathrm{C}$ for $1 \mathrm{~h}$.

5| Remove particulates and unbroken cells with low-speed centrifugation $\left(250 \mathrm{~g}, 4^{\circ} \mathrm{C}, 5 \mathrm{~min}\right)$.

6| Chloroform-methanol (CM) precipitate sample (see Box 1).

7| To each protein pellet, add $300 \mu \mathrm{L} 4 \mathrm{SB}$ with $10 \mathrm{mM}$ NEM. Incubate for $10 \mathrm{~min}$ at $37^{\circ} \mathrm{C}$ with occasional agitation of tube to dissolve pellet.

$\triangle$ CRITICAL STEP The protein denaturation accompanying this step facilitates access of NEM to Cys that are buried within the folded protein interior.

? TROUBLESHOOTING

8| To each tube, add $900 \mu \mathrm{L}$ of LB with $1 \mathrm{mM} \mathrm{NEM}, 1 \times$ PI, $1 \mathrm{mM}$ PMSF, and 0.2\% Triton X-100. Transfer to 1.5-ml screw-cap centrifuge tubes. Incubate overnight at $4{ }^{\circ} \mathrm{C}$ with gentle rocking.

PAUSE POINT Overnight incubation with NEM.

\section{Acyl-biotin exchange reactions}

9| Remove NEM from samples by three sequential CM precipitations. Transfer samples to $12-\mathrm{ml}$ screw-cap tubes and CM precipitate as described in Box 1. After the first two CM precipitations, add $300 \mu \mathrm{L} 4 \mathrm{SB}$ and dissolve protein

\section{BOX 1 | CHLOROFORM-METHANOL PRECIPITATION OF PROTEINS}

Chloroform-methanol (CM) precipitation ${ }^{20}$ is used extensively in this protocol to move proteins between the different chemical treatment steps. The steps below are all done at room temperature (RT).

1. With $1.2 \mathrm{ml}$ of sample per $12-\mathrm{ml}$ screw-cap centrifuge tube, add 4 volumes $(4.8 \mathrm{ml})$ methanol. Vortex to mix.

2. Add 1.5 volumes $(1.8 \mathrm{ml})$ chloroform. (Use glass pipette.) Vortex to mix.

3. Add 3 volumes $(3.6 \mathrm{ml})$ water. Vortex to mix.

4. Centrifuge $\left[6,000 \mathrm{~g}, \mathrm{RT}\left(22-25^{\circ} \mathrm{C}\right), 20 \mathrm{~min}\right]$ in Sorvall HS-4 swinging bucket rotor to separate phases. Should separate into two phases, with protein precipitated at the interphase.

$\triangle$ CRITICAL STEP In fixed-angle rotors, the protein precipitate partially adheres to the side of the tube.

5. Remove and discard top aqueous phase (water and methanol) using Pasteur pipette, taking care not to remove the interphase protein.

6 . Add 3 volumes $(3.6 \mathrm{ml})$ methanol. Mix gently by tube inversion so that pancake-like protein interface is not fully disrupted.

7. Centrifuge $(6,000 \mathrm{~g}, \mathrm{RT}, 10 \mathrm{~min})$ in Sorvall HS-4 swinging bucket rotor. Protein should now precipitate as a loose pellet to the tube bottom.

8. Remove and discard supernatant using Pasteur pipette. Remove any remaining liquid with Pasteur pipette with tip pulled to a fine point.

$\triangle$ CRITICAL STEP Protein is difficult to re-dissolve if methanol and chloroform are not fully removed.

9. Air-dry for 2-3 min. 
pellet by incubating at $37{ }^{\circ} \mathrm{C}$ for 10 min with occasional vortex mixing. Then, dilute with $900 \mu \mathrm{LB}$ containing $0.2 \%$ Triton X-100.

$\triangle$ CRITICAL STEP Residual NEM can greatly reduce biotinylation, irreversibly modifying thiols as they become exposed from palmitoylated Cys by hydroxylamine and thus blocking subsequent reaction with thiol-specific biotinylation reagent.

- PAUSE POINT After first or second CM precipitation, sample may be stored in $4 \mathrm{~S}$ buffer overnight at $-20{ }^{\circ} \mathrm{C}$. The next day, thaw at room temperature $\left(\mathrm{RT} ; 23-25{ }^{\circ} \mathrm{C}\right)$, and dilute with LB.

10| After the third and final CM precipitation, dissolve protein pellet in $250 \mu \mathrm{L} 4 \mathrm{SB}, 37{ }^{\circ} \mathrm{C}, 10 \mathrm{~min}$. At this point the sample is divided into two equal portions (+HA sample and $-\mathrm{HA}$ sample). To ensure that the two samples are equalized, the entire protein sample is pooled into a single tube and then distributed at $240 \mu \mathrm{l}$ per $1.5-\mathrm{ml}$ screw-cap centrifuge tube. +HA samples are diluted fivefold with the addition of $960 \mu \mathrm{l}$ of the hydroxylamine-containing +HA buffer; for - HA samples, $960 \mu \mathrm{l}$ of $-\mathrm{HA}$ buffer is used. Incubate at RT for $1 \mathrm{~h}$ with end-over-end rotation.

11| Transfer samples to $12-\mathrm{ml}$ screw-cap tubes and CM precipitate (Box 1).

12| Dissolve each resulting protein pellet in $240 \mu \mathrm{l}$ 4SB. Dilute with addition of $960 \mu \mathrm{l}$ low-HPDP-biotin buffer. Incubate at RT for $1 \mathrm{~h}$ with end-over-end rotation.

13| To remove unreacted HPDP-biotin before the streptavidin-agarose affinity purification, subject samples to three sequential CM precipitations (Box 1). After the first and second precipitations, dissolve and dilute precipitated proteins as described for Step 10. After the third CM precipitation, dissolve each pellet in $120 \mu \mathrm{L} 2 \mathrm{SB}$ at $37^{\circ} \mathrm{C}, 10 \mathrm{~min}$.

! CAUTION Unremoved HPDP-biotin competes with biotinylated protein for streptavidin-agarose binding.

- PAUSE POINT After the first or second CM precipitation, sample is typically stored in 4 SB overnight at $-20{ }^{\circ} \mathrm{C}$. The next day, thaw at RT, before LB addition.

\section{Affinity purification of biotinylated proteins}

14| Dilute SDS to $0.1 \%$ before adding samples to streptavidin-agarose. For this, first pool like samples (e.g., +HA samples with + HA samples), then dilute 20 -fold with addition of LB containing $0.2 \%$ Triton X-100, $1 \times$ PI and $1 \mathrm{mM}$ PMSF. Aliquot $1 \mathrm{ml}$ per 1.5 -ml screw-cap centrifuge tube and incubate at RT for 30 min with end-over-end rotation.

15| Centrifuge 15,000g (13,000 r.p.m. in the micro-centrifuge) for $1 \mathrm{~min}$ to remove particulates, transferring supernatant to new tubes containing $15 \mu \mathrm{l}$ streptavidin-agarose, pre-equilibrated with LB containing $0.1 \%$ SDS and $0.2 \%$ Triton X-100. Incubate at RT for 90 min with end-over-end rotation.

$\triangle$ CRITICAL STEP Particulates removed by this pre-spin otherwise would pellet along with the streptavidin-agarose through subsequent wash steps and would nonspecifically contaminate the final purified sample.

16 Remove unbound proteins by four sequential 1-ml washes with LB containing $0.1 \%$ SDS and $0.2 \%$ Triton X-100.

17| Release bound proteins from the affinity resin through reduction of the protein-biotin disulfide linkages. For this elution, resuspend the resin in each tube in $150 \mu \mathrm{L}$ LB containing $0.1 \%$ SDS, $0.2 \%$ Triton X-100 and $1 \% \beta$-mercaptoethanol. Incubate for 15 min at $37^{\circ} \mathrm{C}$ with occasional gentle mixing to resuspend settling resin.

18| Pool like eluants together into $1.5-\mathrm{ml}$ screw-cap centrifuge tubes, then concentrate by trichloroacetic acid (TCA) precipitation: add TCA to $10 \%$ (add one-tenth volume of 100\% TCA), incubate $20 \mathrm{~min}$ on ice then collect precipitates by $15,000 \mathrm{~g}, 10 \mathrm{~min}, 4^{\circ} \mathrm{C}$ centrifugation. Dissolve the pellet in $30 \mu \mathrm{L}$ SBB. Then dilute to $150 \mu \mathrm{l}$ with LB.

- PAUSE POINT At this point, samples may be stored at $-80{ }^{\circ} \mathrm{C}$ for up to 1 week.

19| Compare $1 \%$ of the final $+\mathrm{HA}$ and $-\mathrm{HA}$ samples by SDS-polyacrylamide gel electrophoresis (PAGE) and silver-staining (e.g., Fig. 2).

? TROUBLESHOOTING

20| Immediately before sending to MS collaborators, clean up the samples further using two sequential CM precipitations to remove residual detergents. For this, the CM precipitation (described above) is scaled down: with 150- $\mu$ l samples in 1.5-mL screw-cap centrifuge tubes, added methanol, chloroform and water volumes are scaled down proportionately.

21 After the first CM precipitation, dissolve the pellet in $15 \mu \mathrm{L}$ 2SB, then dilute to $150 \mu \mathrm{l}$ with LB. Ship the final 'wet' protein pellet overnight to MS collaborators on dry ice (i.e., to avoid dislodging the pellet from the tube bottom during transport, the sample is not desiccated).

PAUSE POINT At this point, samples may be stored at $-80{ }^{\circ} \mathrm{C}$ for up to 1 month. 


\section{BOX 2 | MULTI-DIMENSIONAL PROTEIN IDENTIFICATION TECHNOLOGY}

The hallmark feature of multi-dimensional protein identification technology (MuDPIT) is its $2 \mathrm{D}$ chromatographic separation of a peptide mixture ${ }^{7,8}$. The microcapillary chromatography column is packed with strong cation exchange (SCX) resin over reversed-phase (RP) resin and is coupled directly to the tandem MS. Over a 10-12 $\mathrm{h}$ time course, peptides are driven through the column by a stepped series of HPLC-driven buffer changes, with eluting peptides being introduced into the tandem MS by electrospray. Each chromatographic step is initiated by a salt bump that drives a subset of the peptides from the $\mathrm{SCX}$ resin onto the RP resin, where they are resolved by their relative hydrophobicity, being eluted from the RP by an increasing gradient of acetonitrile. For details on both setting up and running the microcapillary column chromatography, see ref. 16; specifications for the columns and resins used in our analyses are listed under EQUIPMENT.

\section{Sample proteolysis}

22| Dissolve protein pellets containing 1-20 $\mu \mathrm{g}$ total protein in $50 \mu \mathrm{l}$ proteolysis buffer.

23| Add TCEP to $5 \mathrm{mM}$ and incubate at RT for 30 min to reduce disulfide bonds.

24| Add iodoacetamide to $10 \mathrm{mM}$ and incubate at RT for an additional $30 \mathrm{~min}$ to alkylate free thiols.

25 Add $0.15 \mu \mathrm{g}$ endoproteinase Lys-C (approximate substrate to enzyme mass:mass ratio of 100:1) and digest proteins for $4 \mathrm{~h}$ at $37^{\circ} \mathrm{C}$.

26| Dilute sample fourfold with addition of $100 \mathrm{mM} \mathrm{Tris} / \mathrm{Cl} \mathrm{pH} \mathrm{8.5;} \mathrm{add} \mathrm{CaCl}_{2}$ to $2 \mathrm{mM}$, then $0.5 \mu \mathrm{g}$ trypsin. Incubate overnight $(12-16 \mathrm{~h})$ at $37^{\circ} \mathrm{C}$.

PAUSE POINT Samples may be stored at this point for up to 1 month frozen at $-80{ }^{\circ} \mathrm{C}$.

27| Just before MS/MS analysis, formic acid is added to $5 \%$, and insoluble particulates are removed by centrifugation $(16,000 \mathrm{~g}, 15 \mathrm{~min})$.

\section{MuDPIT chromatography and collection of tandem mass spectra}

28| Refer to Box 2 for information about MuDPIT. Load peptide samples onto desalting column. Desalt with $5 \%$ acetonitrile, $0.1 \%$ formic acid.

29| With peptides still retained, couple the desalting column via the microfilter assembly in-line to the analytical column, which is dually packed with $\mathrm{SCX}$ resin over RP resin.

30| Use the six-step chromatographic program (Box 3 ) to move the peptides from the desalting column onto and through the analytical column with electrospray-mediated elution, and ultimately into the tandem MS. Buffer changes are effected by the HPLC, driven from the tandem MS using Excalibur software.

\section{BOX 3 | MULTI-DIMENSIONAL PROTEIN IDENTIFICATION TECHNOLOGY CHROMATOGRAPHIC PROGRAM}

1. An 80 -min gradient to $45 \%$ buffer B, followed by a 10 -min gradient to $100 \%$ buffer B, then 10 min of buffer B alone. This step elutes peptides from the desalting column to the strong cation exchange (SCX) resin. Peptides that fail to bind to SCX chromatograph through the reversedphase (RP) resin and elute into the tandem MS.

2-5. Initiated with an increasing series of salt bumps to drive charged peptides off the SCX resin onto the C18 resin.

2. First, 3 min of $25 \%$ buffer C, followed by an 80 -min gradient to $45 \%$ buffer B, then a 10 -min gradient to $100 \%$ buffer B and finally 10 min of buffer $\mathrm{B}$ alone.

3. First, 3 min of $50 \%$ buffer C, followed by an 80 -min gradient to $45 \%$ buffer $B$, then a 10 -min gradient to $100 \%$ buffer B and finally 10 min of buffer $\mathrm{B}$ alone.

4. First, 3 min of $75 \%$ buffer C, followed by an 80 -min gradient to $45 \%$ buffer B, then a 10 -min gradient to $100 \%$ buffer B and finally 10 min of buffer $B$ alone.

5. First, 3 min of $100 \%$ buffer C, followed by an 80 -min gradient to $45 \%$ buffer B, then a 10 -min gradient to $100 \%$ buffer B and finally 10 min of buffer $\mathrm{B}$ alone.

6. First, 20 min of $100 \%$ buffer C, followed by a 90 -min gradient to $45 \%$ buffer B, then a 10 -min gradient to $100 \%$ buffer B and finally 10 min of buffer $\mathrm{B}$ alone.

Flow rate through the column is maintained at a constant $150 \mathrm{nl} \mathrm{min}{ }^{-1}$. Eluting peptides are introduced into the tandem MS by electrospray at a distally applied spray voltage of $2.4 \mathrm{kV}$. Column eluate is continuously analyzed by MS/MS over the six-step chromatography program. One full-range mass-scan $(400-1,600 \mathrm{~m} / \mathrm{z})$ should be followed by the collection of seven data-dependent tandem mass spectra at a $35 \%$ collision energy in a continuous loop. 
PROTOCOL

\section{Data analysis: protein identifications}

31| MS/MS spectra are analyzed on an Intel Xenon 80-processor cluster running under the Linux operating system. Use PARC ${ }^{17}$ to analyze charge state and filter data quality of MS/MS spectra.

32| Search relevant protein database, supplemented with common contaminants (e.g., keratins) using SEQUEST ${ }^{18}$.

33| Filter SEQUEST results using DTASelect v1.919 with the following parameters:

Minimum correlation coefficients of 1.8, 2.5 and 3.5, for singly, doubly and triply charged precursor ions, respectively

Minimum $\Delta \mathrm{C}_{n}$ of 0.08

Minimum requirement of two peptides per run

\section{Spectral count-based quantitation}

34| Extract spectral counts from DTASelect MS/MS datafiles. Open DTASelect datafile (in .txt format) using Excel. Data fields sort into the rows and columns of the worksheet.

35 Collect DTASelect summary lines. Heading the data for each identified protein within the DTASelect datafile is a summary line reporting various information, including the protein's unique database ID, its database annotation, its predicted molecular weight and pI, as well as a summary of the MuDPIT data associated with the identification (including the spectral count). DTASelect summary lines are marked at the left with the identifier " $U$," which should sort to the leftmost column (column A) of the Excel worksheet. "Sort" worksheet on the basis of column A in "descending" order. Summary lines should now be collected near the top of the worksheet (Fig. 5a). Delete all rows that do not contain the "U" identifier, conserving just the summary line for each identified protein.

36| Delete all columns containing extraneous information (Fig. 5b).

37| Use the Excel "consolidate" function to combine spectral count datasets from the MuDPIT runs under comparison onto a single spreadsheet (Fig. 5c).

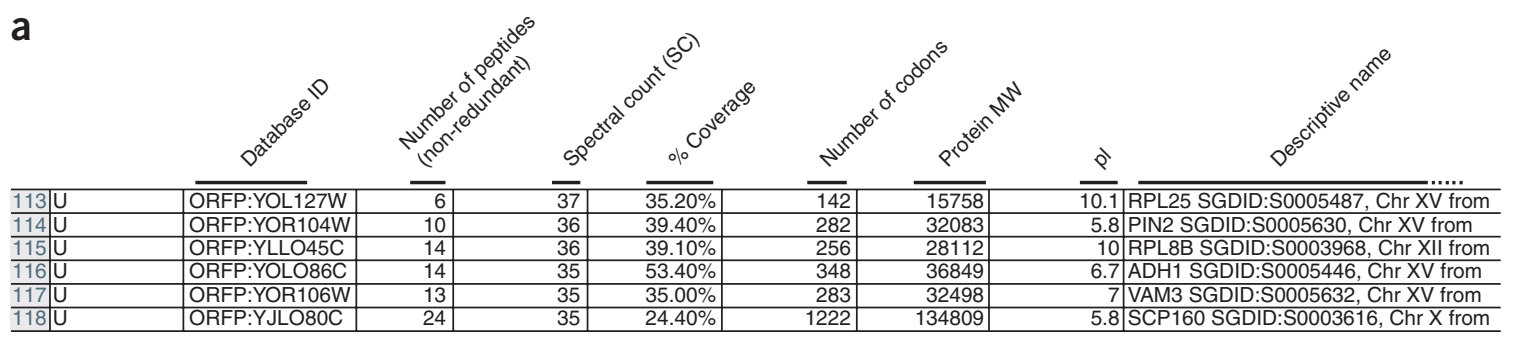

b

\begin{tabular}{c|c|c|c}
\multicolumn{2}{c}{ ID } & \multicolumn{3}{c}{ SC } \\
\cline { 2 - 4 } 113 & YOL127W & 37 & \\
\hline 114 & YOR104W & 36 & \\
\hline 115 & YLLO45C & 36 & \\
\hline 116 & YOLO86C & 35 & \\
\hline 117 & YOR106W & 35 & \\
\hline 118 & YJLO80C & 35 & \\
\hline
\end{tabular}

C

\begin{tabular}{|c|c|c|c|}
\hline \multirow[t]{2}{*}{ 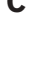 } & \multirow[b]{2}{*}{ ID } & \\
\hline & & $+\mathrm{HA}$ & $-\mathrm{HA}$ \\
\hline 113 & YOL127W & 37 & 31 \\
\hline 114 & YOR104W & 36 & 0 \\
\hline 115 & YLLO45C & 36 & 19 \\
\hline 116 & YOLO86C & 35 & 62 \\
\hline 117 & YOR106W & 35 & 2 \\
\hline 118 & YJLO80C & 35 & 42 \\
\hline
\end{tabular}

d

\begin{tabular}{|c|c|c|}
\hline d & & \\
\hline ID & $+\mathrm{HA}$ & $-\mathrm{HA}$ \\
\hline \begin{tabular}{l|l|}
13 & YOL127W \\
\end{tabular} & 31.7 & 41.2 \\
\hline \begin{tabular}{l|l}
14 & YOR104W \\
\end{tabular} & 30.9 & 0 \\
\hline \begin{tabular}{l|l}
15 & YLLO45C \\
\end{tabular} & 30.9 & 25.2 \\
\hline \begin{tabular}{|l|l|}
116 & YOLO 86C \\
\end{tabular} & 30 & 82.4 \\
\hline \begin{tabular}{l|l|}
117 & YOR106W \\
\end{tabular} & 30 & 2.7 \\
\hline \begin{tabular}{|l|l|}
$118 J L O 80 C$ \\
\end{tabular} & 30 & 55.8 \\
\hline
\end{tabular}

e

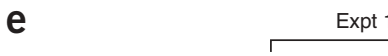

\begin{abstract}
Expt 2
\end{abstract}

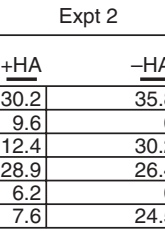

Avg (1\&2)

\begin{tabular}{|c|c|}
\hline $\mathrm{HA}$ & - \\
\hline$\overline{|c|}$ & 38.5 \\
\hline .2 & 0 \\
\hline \begin{tabular}{l|l}
.6 \\
\end{tabular} & 27.7 \\
\hline 94 & 54.4 \\
\hline \begin{tabular}{l|l}
3.1 \\
\end{tabular} & 1.4 \\
\hline \begin{tabular}{l|l}
3.8 &
\end{tabular} & 40.2 \\
\hline
\end{tabular}

f

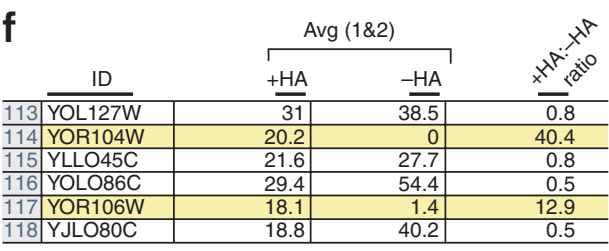

Figure 5 | Example spectral count analysis. Analysis is shown for an arbitrary set of five proteins of similar mid-abundance range identified from published analysis of yeast acyl-biotinyl exchange (ABE) samples ${ }^{4}$. (a) DTASelect summary lines. After the DTASelect .html file [from multi-dimensional protein identification technology (MuDPIT) of a yeast plus-hydroxylamine (HA) sample] is pasted in its entirety into an Excel spreadsheet, the "Sort" command is used to collect summary lines that are uniquely identified by the " $U$ " in the leftmost column. All other rows are deleted. (b) Extraction of spectral count data. Delete all columns except for the ones containing database IDs and spectral counts. (c) "Consolidate" spectral count data from MuDPIT runs under comparison. (d) Normalize data. Each spectral counts is divided by the total spectral count for the sample (strategy 1 of Box 4; 17,494 for the +HA run and 11,293 for the -HA run) then multiplied by the arbitrary multiplier 15,000. (e) Average normalized spectral count data from like experiments. The data from experiment 1 are assembled (using the "Consolidate" command) with data, manipulated as above, from experiment 2. Normalized spectral counts from the two experiments are averaged. (f) + HA: - HA ratios identify candidate PPs. Before dividing averaged and normalized + HA spectral counts by the averaged and normalized - HA spectral counts, averaged and normalized -HA spectral counts of zero are arbitrarily replaced by a value of 0.5 (to avoid division by zero). The highlighted proteins encoded by YDR104W and YOR106W (corresponding to Pin2 and Vam3, respectively) are identified as candidate PPs. 


\section{BOX 4 | NORMALIZATION STRATEGIES}

Strategy 1 normalizes the spectral count data on the basis of the total spectral count yields. This method makes the assumption that in each multi-dimensional protein identification technology (MuDPIT) sample run, peptide identifications are being made at or near the capacity of the tandem MS instrument, which is generally a good assumption for complex samples comprising $10 \mu \mathrm{g}$ or more of total protein. For data formatted by DTASelect v.1.9, the total spectral count yield is reported in the final summary table as the number of redundant peptide IDs. To normalize, generate a new Excel spreadsheet column that divides the spectral count number associated with each identified protein by the total spectral count yield for that sample and then multiplies each by some arbitrary constant, reflective of the approximate average total spectral count yield, e.g., 10,000 .

Strategy 1 was used in our published yeast analysis, both for plus- and minus-hydroxylamine (HA) sample comparisons and for the mappings of palmitoyl protein (PP) substrates to the DHHC protein acyl transferase (PAT) enzymes, in this case comparing $+\mathrm{HA}$ samples of wild-type and DHHC gene-deleted yeast strains ${ }^{4}$. However, it has proved less satisfactory in our more recent analyses of samples from neurons (data not shown). Palmitoylation appears to be a much more prominent protein modification in neurons than in yeast and, consequently, acyl-biotinyl exchange (ABE)-processed - HA neuronal samples show substantially reduced protein complexity relative to the parallel $+\mathrm{HA}$ samples. Thus, our neuronal analyses have relied on an improved strategy:

Strategy 2 utilizes abundant proteins from the contaminant protein background common to both $+\mathrm{HA}$ and $-\mathrm{HA}$ samples for normalization. For instance, $\alpha$-tubulin is an abundant protein contaminant that appears to contaminate both $\mathrm{ABE}$-purified $+\mathrm{HA}$ and $-\mathrm{HA}$ samples from neurons as well as from yeast equivalently. Raw spectral count numbers associated with $\alpha$-tubulin typically ranged between 200 and 700 counts per run. To normalize spectral counts, a multiplier calculated to equalize $\alpha$-tubulin counts is applied to all the identified proteins. For instance, if 300 spectral counts were associated with $\alpha$-tubulin in sample 1 and 600 in sample 2, then normalization is achieved by multiplying the spectral counts associated with each sample 1 protein by a factor of 2.0 .

38| Normalization of spectral count data (Fig. $5 \mathrm{~d}$ and Box 4). Unavoidable variation introduced at the level of the ABE purification and/or the running of the MuDPIT 2D chromatography results in spectral count yields that may vary by as much as twofold to threefold for identical proteins in equivalent samples. Despite these differences in overall spectral count yields, the spectral count ranking of individual identified proteins, particularly for the more abundant sample proteins identified by high spectral count numbers, typically is very well reproduced. Two normalization strategies have been employed to accommodate sample-to-sample spectral count yield variation (see Box 4).

39| Average like data from iterative MuDPIT analyses. Spectral count numbers are statistics, and thus confidence in conclusions derived from spectral count data is increased through repeat analysis. Our identification of yeast PPs was based on the analysis of four paired $+\mathrm{HA}$ and $-\mathrm{HA}$ samples ${ }^{4}$. Generate a new Excel spreadsheet column that averages the normalized spectral counts for equivalent samples-e.g., a column reporting averaged spectral count numbers from + HA samples and a column reporting averaged - HA spectral count numbers (Fig. 5e).

40| Compare the abundance level of each protein within the different samples through ratiometric comparisons of averaged and normalized spectral count data (Fig. 5f). Candidate PPs are identified, for instance, by comparing each protein's + HA sample representation with its $-\mathrm{HA}$ sample representation (PPs show higher $+\mathrm{HA}$ sample abundance).

41| Generate a new spreadsheet column that divides normalized and averaged counts for each identified protein from the $+\mathrm{HA}$ samples by the normalized, averaged counts from the $-\mathrm{HA}$ samples. Before ratio calculations, to avoid division by 0 , replace zeros present in the divisor column with some small arbitrary number (choose a number somewhat smaller than the smallest value present in divisor column). Such replacement is easily automated with the Excel "IF" function. Candidate PPs are identified by their high $+\mathrm{HA}:-\mathrm{HA}$ spectral count ratios.

42| Graphically compare spectral count data using either $x, y$-scatter plots (see Box 5) or colorimetric depictions of the ratios (see Box 6).

- TIMING

From start to finish, approximately 2 weeks are required.

Day 1-Steps 1-8, extract preparation, culiminating in overnight NEM blockade: 4-6 h

Day 2-Steps 9-13, acyl-biotin exchange: $8 \mathrm{~h}$

Day 3-Steps 14-18, streptavidin-agarose affinity purification: $8 \mathrm{~h}$

Day 4-Steps 19-21, SDS-PAGE analysis of purified samples: $8 \mathrm{~h}$

Day 5-Steps 22-27, sample proteolysis: $16 \mathrm{~h}$

Days 6 and 7-Steps 28-30, column preparation, loading, and overnight MuDPIT analysis: $18 \mathrm{~h}$

Days 8-10-Steps 31-33, computer analysis of tandem MS: 48-72 h (depending on computing power)

Day 11-Steps 34-42, spectral count analysis: $4 \mathrm{~h}$ 


\section{BOX $5 \mid X, Y$-SCATTER PLOTS}

Each identified protein is plotted using the averaged, normalized spectral counts as $x$ - and $y$-coordinates. For instance, Figure 3 reports the multi-dimensional protein identification technology (MuDPIT) data from our recent analysis of palmitoylation in yeast ${ }^{4}$. The 1,557 proteins identified from MuDPIT analysis of three paired plus- and minus-hydroxylamine (HA) samples are displayed: each individual protein is represented by a dot plotted as the averaged, normalized $+\mathrm{HA}$ sample spectral count ( $x$-coordinate) against the averaged, normalized $-\mathrm{HA}$ sample spectral count ( $y$-coordinate). The palmitoyl proteins (PPs) are shown as red dots; these correspond both to the 15 PPs that were known at the outset of this analysis to be palmitoylated and to the 35 new PPs identified and confirmed by this analysis ${ }^{4}$. The PPs clearly stand out as clustering at or near the $x$-axis. In contrast, contaminant proteins, which are equivalently purified into both the $+\mathrm{HA}$ and $-\mathrm{HA}$ samples, tend to fall near the $x, y$-diagonal. This graphical approach also gives a sense of the statistical significance of candidate PP identifications; note that at low spectral count numbers the PP cluster begins to merge and overlap the $x, y$-diagonal contaminant protein cluster. In addition to its utility in identifying candidate PPs, the $x, y$-scatter plot approach should prove useful in other binary comparisons, particularly for highlighting PPs that are affected by palmitoylation-perturbing conditions (e.g., plus and minus drug treatment).

\section{? TROUBLESHOOTING}

Troubleshooting advice can be found in Table 1.

TABLE 1 | Troubleshooting table.

\begin{tabular}{llll}
\hline Step & Problem & Possible reasons & Solution \\
\hline 7 & Protein fails to fully dissolve in 4\% & Too much protein & $\begin{array}{l}\text { Increase 4SB volume as well as the number } \\
\text { of tubes used in processing each sample }\end{array}$
\end{tabular}

19 Indistinguishable gel profiles for proteins purifed into plus- and minus-hydroxylamine (HA) samples
Palmitoyl modifications lost either before or during purification

Insufficient $N$-ethylmaleimide (NEM) blockade of free thiols

Residual NEM may be blocking thiols as they become exposed by hydroxylamine

Failure to release biotinylated proteins from streptavidin-agarose
Maintain tissues or cells in cold before purification

Check buffer $\mathrm{pH}$ (thioesters are most stable at neutral $\mathrm{pH}$ )

Increase NEM concentration in Step 8

Change buffering agent of lysis buffer (LB) used in Steps 1-7. In addition to reacting with thiols, NEM also reacts with primary amines at low level. Thus, Tris may be consuming NEM. Substitute HEPES or phosphate buffer for Tris in LB

Increase number of chloroform-methanol precipitations

Increase $\beta$-mercaptoethanol concentration Try $50 \mathrm{mM}$ DTT

Non-specific streptavidin-agarose binding

Reduce amount of streptavidin-agarose used

\section{ANTICIPATED RESULTS}

PPs should be substantially over-represented in $+\mathrm{HA}$ versus $-\mathrm{HA}$ samples and thus should be highlighted by the ratiometric comparison of $+\mathrm{HA}$ and $-\mathrm{HA}$ sample spectral counts for each identified protein. For abundant sample proteins (i.e., those identified by high spectral count numbers), the bona fide PPs should be cleanly resolved from the background of co-purifying contaminant proteins. Contaminant proteins tend to be proteins of known high abundance, e.g., cytoskeletal proteins, chaperones, ribosomal proteins and glycolytic enzymes, that generally are purified in hydroxylamine-independent fashion and thus show plus-to-minus spectral count ratios near 1. At lower spectral counts, distinguishing PPs from contaminants becomes a little more problematic; at these low numbers, some non-palmitoylated contaminant proteins show skewed plus-to-minus spectral count ratios owing purely to chance.

\section{Spectral count statistical variation}

Spectral counts correlate with, but do not directly measure, abundance ${ }^{10}$. For proteins identified by low spectral count numbers, substantial run-to-run spectral count variation may be seen in parallel MuDPIT runs of identical samples, a reflection, 


\section{BOX 6 | COLORIMETRIC DEPICTIONS OF RATIOS}

This approach, extrapolated from the genomic analysis of microarray data, when applied to spectral count data, allows sample-to-sample changes in individual protein abundance to be depicted. Figure 4 is a colorimetric depiction of the relative abundance of the top 30 yeast palmitoyl proteins (PPs) in plus-hydroxylamine $(+\mathrm{HA})$ samples from wild-type yeast versus a series of mutant yeast strains deficient for members of the DHHC family of protein acyl transferases (PATs) $)^{4}$. The different strains have the DHHC PATs mutated either individually or in combination (Fig. 4; strain genotypes are indicated to the left). For each protein, the ratio of its wild-type sample spectral counts to its mutant sample spectral counts is mapped onto a red-green colorimetric gradient where red corresponds to proteins with 20-fold or greater spectral count under-representations in the purified mutant strain $+\mathrm{HA}$ acyl-biotinyl exchange (ABE) samples relative to equivalent wild-type samples. A green coloration would indicate mutant strain over-representation (Fig. 4; as one would expect, none of the proteins show substantial mutant strain over-representations). Black, the absence of green or red, indicates proteins with no appreciable abundance change between wild-type and mutant strain samples. Intermediate ratios, reflective of intermediate mutant strain sample under- or over-representations, are reported by intermediate red or green shadings. The Figure 4 heat map provides both a sense of the overall participation of the DHHC PAT family in palmitoylation and a first sense of DHHC PAT specificity, yielding a first crude enzyme-substrate map that links the individual PPs to their cognate, modifying PAT. Similar to the $x, y$-scatter plot, this approach should prove useful in identifying the specific changes in palmitoylation brought on by a variety of perturbants.

presumably, of variations in both the peptidyl chromatography and peptide sampling by the tandem MS. Ultimately, spectral count is a statistic, and confidence in a result is reinforced by repeat analyses and also, perhaps, by statistical tests of significance. For our analyses to date, we have tended to derive conclusions mainly from the set of proteins that are identified by high spectral count numbers. For instance, our mapping of PP substrates with DHHC PATs (Fig. 5), which relied on comparing spectral count scores from $+\mathrm{HA}$ samples derived from DHHC PAT mutant yeast strains with scores from wild-type yeast +HA samples, focused on just the 30 top-scoring yeast PPs. Spectral count reductions of tenfold or more specific to the DHHC mutant strain samples easily highlighted the links between substrate PPs and DHHC PATs. Confidence in detected palmitoylation changes in the two- to fivefold range may require repeat analyses coupled with statistical tests of significance.

\section{False positives}

Two classes of false-positive proteins typically are seen. One class, 'statistical false-positives', result from the spectral count statistical variation discussed above, i.e., proteins that show high plus-to-minus spectral count ratios simply owing to chance under-detection from the $-\mathrm{HA}$ samples. Such false positives become increasingly dominant as one proceeds further down the list to proteins identified by low spectral count numbers. Repetition of the MuDPIT analysis reduces this statistical noise. A second class of false positives comprises proteins that are not palmitoylated, but that are strongly and specifically detected by $A B E$ none the less. As ABE detects PPs through detection of the palmitoyl-cysteinyl thioester linkage, it is not surprising that some proteins that utilize thioesters for chemistries other than palmitoylation also are purified by ABE. Two examples of this class, prominently detected by our yeast analysis, are Pdx1 and Lat1, which are both subunits of the mitochondrial pyruvate dehydrogenase complex. In the decarboxylation of pyruvate, these two subunits transiently accept acetyl moieties in thioester linkage to lipoic acid prosthetic groups. Although strongly detected by ABE, Pdx1 is not palmitoylated and it is not labeled in experiments that assess palmitoylation through metabolic $\left[{ }^{3} \mathrm{H}\right]$ palmitic acid incorporation ${ }^{4}$. Other thioester-utilizing false positives detected in our yeast proteomic analysis include Gcv3, which uses the lipoic acid prosthetic group for glycine decarboxylation, the E2 ubiquitin conjugase Ubc1, which transiently accepts ubiquitin moieties in thioester linkage, and the acyl-carrier protein Acp1, which carries growing fatty acyl chains in thioester linkage to a phosphopantetheinyl prosthetic group. An orthologous set of thioester-utilizing false positives also are prominently detected in mammalian analyses (R. Kang, J.W., J. Yates, N.G.D. and A. El-Husseini, unpublished results). These thioester-utilizing false positives are always seen; indeed, their detection provides some measure of the efficacy of the ABE reactions and purifications. Furthermore, as their ABE labeling is palmitoylation independent, these proteins should remain unchanged in analyses aimed at the global palmitoylation effects of perturbants, e.g., mutation or drugs, and may prove useful as a standard for normalizing sample-to-sample spectral counts

(Box 4). Particularly useful in this regard are two strongly detected lipoic acid-utilizing subunits of the mitochondrial pyruvate dehydrogenase complex, namely, the dihydrolipoamide $S$-acetytransferase and the lipoyl-containing component $X$ (in yeast, Lat 1 and $\mathrm{Pdx} 1$, respectively).

\section{Candidate testing}

To confirm palmitoylation and to eliminate false positives, the newly identified PP candidates should be independently tested for palmitoylation. Statistical false positives can be eliminated in small-scale experiments that use ABE chemistry to detect palmitoylation. Such a scaled-down ABE protocol involves a scaled-down ABE processing of extracts and immunoprecipitation of an epitope-tagged version of the protein under consideration, with a final western blot detection using anti-biotin antibodies 4 . 
PROTOCOL

However, the alternative approach, the direct testing of the candidate protein incorporation of a label from $\left[{ }^{3} \mathrm{H}\right]$ palmitic acid via classic metabolic labeling ${ }^{4}$, may be preferred: it has the added advantage of eliminating the thioester-utilizing class of false positives.

ACKNOWLEDGMENTS We thank John R. Yates 3rd (The Scripps Research Institute, La Jolla, CA) for his help in the original development of this protocol. The MuDPIT proteomic technology developed by his laboratory proved indispensable to this analysis in terms of its high capacity and the facile quantitative analysis that it affords. The development of this technology was supported by NIH GM065525 (N.G.D.) and NIH RR11823 (John R. Yates 3rd).

COMPETING INTERESTS STATEMENT The authors declare no competing financial interests.

Published online at http://www.natureprotocols.com

Rights and permissions information is available online at http://npg.nature.com/ reprintsandpermissions

1. Huang, K. \& El-Husseini, A. Modulation of neuronal protein trafficking and function by palmitoylation. Curr. Opin. Neurobiol. 15, 527-535 (2005).

2. Resh, M.D. Palmitoylation of ligands, receptors, and intracellular signaling molecules. Sci. STKE 2006, re14 (2006).

3. Linder, M.E. \& Deschenes, R.J. Palmitoylation: policing protein stability and traffic. Nat. Rev. Mol. Cell Biol. 8, 74-84 (2007).

4. Roth, A.F. et al. Global analysis of protein palmitoylation in yeast. Cell 125, 1003-1013 (2006).

5. Roth, A.F., Wan, J., Green, W.N., Yates, J.R. \& Davis, N.G. Proteomic identification of palmitoylated proteins. Methods 40, 135 (2006).

6. Drisdel, R.C. \& Green, W.N. Labeling and quantifying sites of protein palmitoylation. Biotechniques 36, 276-285 (2004).

7. Link, A.J. et al. Direct analysis of protein complexes using mass spectrometry. Nat. Biotechnol. 17, 676-682 (1999).

8. Washburn, M.P., Wolters, D. \& Yates, J.R. 3rd. Large-scale analysis of the yeast proteome by multidimensional protein identification technology. Nat. Biotechnol. 19, 242-247 (2001).

9. Aebersold, R. \& Mann, M. Mass spectrometry-based proteomics. Nature 422, 198-207 (2003).
10. Liu, H., Sadygov, R.G. \& Yates, J.R. 3rd. A model for random sampling and estimation of relative protein abundance in shotgun proteomics. Anal. Chem. 76, 4193-4201 (2004).

11. Ghaemmaghami, S. et al. Global analysis of protein expression in yeast. Nature 425, 737-741 (2003).

12. Roth, A.F., Feng, Y., Chen, L. \& Davis, N.G. The yeast DHHC cysteine-rich domain protein Akr1p is a palmitoyl transferase. J. Cell Biol. 159, 23-28 (2002).

13. Lobo, S., Greentree, W.K., Linder, M.E. \& Deschenes, R.J. Identification of a Ras palmitoyltransferase in Saccharomyces cerevisiae. J. Biol. Chem. 277, 41268-41273 (2002).

14. Smotrys, J.E. \& Linder, M.E. Palmitoylation of intracellular signaling proteins: regulation and function. Annu. Rev. Biochem. 73, 559-587 (2004).

15. Ducker, C.E. et al. Discovery and characterization of inhibitors of human palmitoyl acyltransferases. Mol. Cancer Ther. 5, 1647-1659 (2006).

16. Link, A.J., Jennings, J.L. \& Washburn, M.P. Analysis of protein composition using multidimensional chromatography and mass spectrometry. in Current Protocols in Protein Science, (eds. Coligan, J.E., Dunn, B.M., Speicher, D.W. \& Wingfield, P.T.) chapter 23, 23.1.1-23.1.25 (John Wiley and Sons, Inc., Hoboken, New Jersey, 2003).

17. Bern, M., Goldberg, D., McDonald, W.H. \& Yates, J.R. 3rd. Automatic quality assessment of peptide tandem mass spectra. Bioinformatics 20 (Suppl 1): I49-I54 (2004).

18. Eng, J.K., McCormack, A.L. \& Yates, J.R. 3rd. An approach to correlate tandem mass spectral data of peptides with amino acid sequences in a protein database. J. Am. Soc. Mass Spectrom. 5, 976-989 (1994).

19. Tabb, D.L., McDonald, W.H. \& Yates, J.R. 3rd. DTASelect and contrast: tools for assembling and comparing protein identifications from shotgun proteomics. J. Proteome Res. 1, 21-26 (2002).

20. Wessel, D. \& Flugge, U.I. A method for the quantitative recovery of protein in dilute solution in the presence of detergents and lipids. Anal. Biochem. 138, 141-143 (1984). 